\title{
Comparing Different Approaches to Model Error Modeling in Robust Identification
}

\author{
Wolfgang Reinelt\#, Andrea Garulli* and Lennart Ljung\# \\ \# Department of Electrical Engineering \\ Linköping University, 58183 Linköping, Sweden \\ WWW: http://www.control.isy.liu.se/ \\ E-mail: \{wolle,ljung\}@isy.liu.se \\ * Dipartimento di Ingegneria dell'Informazione \\ Università di Siena, 53100 Siena, Italy \\ WWW: http://www-dii.ing.unisi.it/〜garulli/ \\ E-mail: garulli@ing.unisi.it
}

August 2, 2000

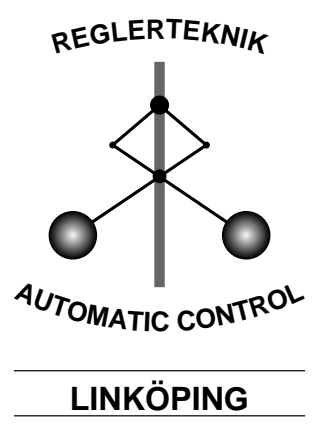

Report no.: LiTH-ISY-R-2293

Prepared for Journal Publication

Technical reports from the Automatic Control group in Linköping are available by anonymous $\mathrm{ftp}$ at the address ftp.control.isy.liu.se. This report is contained in the portable document format file XXXX.pdf. 


\title{
Comparing Different Approaches to Model Error Modeling in Robust Identification*
}

\author{
Wolfgang Reinelt $\#, \dagger$, Andrea Garulli $\# \#$ and Lennart Ljung $\#$ \\ \#Department of Electrical Engineering, \\ Linköping University, 58183 Linköping, Sweden. \\ E-mail: \{wolle,ljung\}@isy.liu.se \\ \#\# Dipartimento di Ingegneria dell'Informazione, \\ Università di Siena, 53100 Siena, Italy. \\ E-mail: garulli@ing.unisi.it
}

\begin{abstract}
Identification for robust control must deliver not only a nominal model, but also a reliable estimate of the uncertainty associated with the model. This paper addresses recent approaches to robust identification, that explicitly aim at separating contributions from the two main uncertainty sources: unmodeled dynamics and noise affecting the data. In particular, the following methods are considered: non-stationary Stochastic Embedding, Model Error Modeling based on prediction error methods, Set Membership Identification. Model validation issues are also addressed in the proposed framework. Moreover, it is shown how the computation of the minimum noise bound for which a nominal model is not falsified by i/o data, can be used as a rationale for selecting an appropriate model class in the set membership setting. For all methods, uncertainty is evaluated in terms of the frequency response, so that it can be handled by $\mathcal{H}_{\infty}$ control techniques. An example, where a nontrivial undermodeling is ensured by the presence of a nonlinearity in the system generating the data, is presented to compare the different methods.
\end{abstract}

Keywords: identification for robust control, model error modeling, unmodeled dynamics, model validation, set membership estimation, stochastic embedding.

\footnotetext{
*Preliminary versions of this work have been presented at the 1999 Conference on Decision and Control, Phoenix, AZ, USA [19] and the IFAC Symposium System Identification SYSID2000, Santa Barbara, CA, USA [6].

${ }^{\dagger}$ Supported by the European Union within the European Research Network in System Identification (ERNSI) under contract number ERB FMRX CT98 0206, which is gratefully acknowledged.
} 


\section{Introduction and Motivation}

One of the main objectives of control-oriented identification is to estimate models that are suitable for robust control design techniques. For this purpose, the identification procedure must deliver not only a nominal model, but also a reliable estimate of the uncertainty associated to the model. Different paradigms for the description of uncertainty have been addressed in the literature (see e.g. the special issues $[13,20]$ and the recent book [7]). Two main philosophies are basically adopted. The first one is based on statistical assumptions and usually leads to least squares estimation techniques and prediction error methods, see [16]. The second one relies on deterministic hypotheses, such as the identification error being unknown-but-bounded (UBB), and has given rise to a number of techniques usually addressed as bounded error or set membership identification (see $[18,17]$ and the references therein).

In standard identification problems, the error originates from two different sources: a "variance" term, due to noise affecting the data, and a "bias" term, due to system dynamics which is not captured by the estimated nominal model (often addressed also as the model error). Clearly, the nature of these two error terms is quite different: the former is generally uncorrelated with the input signal (when the data is collected in open loop), while the latter strongly depends on the estimated nominal model and on the input used in the identification experiment. The model error is not negligible in most practical situations, especially those in which the order of the nominal model must be small (a typical requirement of robust design techniques). Moreover, while a priori information on measurement noise are often available, similar hypotheses on the unmodeled dynamics seem to be less realistic.

This paper addresses three different approaches to robust identification: Stochastic Embedding, Model Error Modeling and Set Membership Identification. Each of them explicitly separate contributions from the above two error sources, thus providing a reliable estimate of model uncertainty. The first two approaches have been developed in the statistical framework, while the latter relies on UBB error assumptions.

Stochastic Embedding $[12,10]$ is a frequency domain method which assumes that unmodeled dynamics can be represented adequately by a non-stationary stochastic process whose variance increases with frequency. The nominal model is obtained via least-squares estimation from frequency domain data; therefore, harmonic inputs are required. The uncertainty associated to the model is evaluated from statistical properties of the random walk process describing the unmodeled dynamics.

Model Error Modeling [14, 15] employs standard prediction error methods to identify a nominal model from input-output time domain data [16]. Then, one can estimate the unmodeled dynamics by looking at that part of the identification residual that originates from the input. Identification of residual dynamics (that can be performed using again prediction error methods) provides the so called model error model. The confidence region of model error model allows one to evaluate the uncertainty associated to the nominal model and can 
be used as a powerful model validation tool.

Set Membership Identification provides efficient algorithms for estimating the set of feasible models, compatible with the available data and the UBB error assumption. The choice of the nominal model is usually performed by minimizing a cost function related to the feasible set. The feasible set itself gives the size of the uncertainty associated to the nominal model. In the first works on set membership identification (see the survey [25] and the references therein) the contributions from unmodeled dynamics and noise were not separated (which corresponds to assuming that the plant generating the data belongs to the considered model class). More recently, the presence of model errors has been explicitly accounted for in several works and different settings (see e.g. $[24,9,8]$ ). In this paper, model error modeling concepts are extended to set membership identification, in order to obtain a fairly general strategy for separating error contributions.

As it can be seen from the above discussion, quite different approaches to robust identification are provided by the considered techniques, the main differences concerning the type of data required, the selected model class, the assumptions on the noise affecting the data and the nominal model estimation criteria. The main contribution of the paper is to test the above three approaches on the same simulation setting, in order to highlight the advantages of each method and to provide, as long as it is possible, a fair comparison of the results. The most important features of each technique are emphasized. In order to ensure the presence of a nontrivial undermodeling, a nonlinearity is present in the true system considered in the test example, and identification of a low order nominal model is required. Frequency domain uncertainty regions are considered in order to provide adequate models for $\mathcal{H}_{\infty}$ control design.

The second contribution of the paper is to provide a frequency domain model validation tool for set membership identification. The basic idea is to evaluate the minimum value of the error bound, which allows one to validate the estimated nominal model. It is also shown that this provides a useful rationale for the selection of an adequate model class.

Paper outline: Section 2 briefly reviews the technique of non-stationary stochastic embedding, while in Section 3, model error modeling ideas are summarized from a quite general point of view. Section 4 introduces the concept of set membership identification, embedded in a model error modeling setup, and discusses identification of both nominal model and model error, model validation and model class selection. Section 5 reports a comparative simulation example, which illustrates the main features of non-stationary stochastic embedding and model error modeling, the latter using either prediction error methods or the proposed set membership identification approach. Benefits and advantages of the three techniques are discussed. Finally, Section 6 gives some concluding remarks and suggests directions of future work. 


\section{Non-Stationary Stochastic Embedding}

The basic idea behind Stochastic Embedding can be described as follows. The true system G can be given as

$$
\mathrm{G}(i \omega)=\hat{\mathrm{G}}_{0}(i \omega)+\Delta \mathrm{G}(i \omega)
$$

where $\hat{G}_{0}$ is a "nominal system" that can be exactly represented within a parameterized family, and $\Delta \mathrm{G}(i \omega)$ is a random variable independent of $\hat{\mathrm{G}}_{0}$. Notice that this is a turn-around of the conventional interpretation, where the nominal, estimated model

$$
\hat{G}_{0}(i \omega)=G(i \omega)+\Delta G(i \omega)
$$

is seen as the true system plus a model error $\Delta \mathrm{G}$, that is independent of $\mathrm{G}$. In contrast, the true system in (1) is a random variable as well, which is the root of the term "stochastic embedding". Now, suppose that we have noisy observations of the true system $\mathrm{G}$ at certain frequencies:

$$
\widehat{\hat{G}}_{k}=G\left(i \omega_{k}\right)+v_{k}
$$

where the noise term $v_{k}$ is independent of $G$ and $\Delta G$. Then, combining (1) and (2), one has

$$
\widehat{\hat{G}}_{k}=\hat{\mathrm{G}}_{0}\left(i \omega_{k}\right)+v_{k}+\Delta \mathrm{G}\left(i \omega_{k}\right) .
$$

This is the basic estimation equation, that needs to be complemented with a parameterization of the model $\hat{G}_{0}$ and assumptions about the variance of $v_{k}$ and $\Delta G\left(i \omega_{k}\right)$. In [10] it is suggested to use a linear regression parameterization of the nominal model in terms of some orthonormal basis functions, i.e. $\hat{G}_{0}(\theta)=\sum_{i=1}^{n} \theta_{i} B_{i}$, where $\mathcal{B}:=\left[B_{1}, \ldots, B_{n}\right]$ represents the selected basis and $\theta$ is the vector of parameters. Moreover, the model error $\Delta \mathrm{G}$ is parameterized according to the same basis $\mathcal{B}$ and it is assumed that the relative model error has a variance that increases linearly or quadratically with $\omega$. This means that $\Delta G$ can be written as $\mathcal{B} \bar{\theta} \Lambda$ where $\Lambda$ is a random walk process over $\omega$ (this argument being suppressed) and $\bar{\theta}$ comes from prior knowledge. All this leads to

$$
\begin{aligned}
\mathrm{G} & =\hat{\mathrm{G}}_{0}(\theta)+\mathrm{G}_{0}(\bar{\theta}) \Lambda \\
& =\mathcal{B} \theta+\mathcal{B} \bar{\theta} \Lambda .
\end{aligned}
$$

The identification procedure then consists of three main steps:

1. Pointwise least squares estimation of the transfer function for certain frequencies: therefore, the input $u$ has to be a sum of sinusoids. This step delivers the values $\hat{\mathrm{G}}_{k}$, for certain frequencies. Additionally, statistical properties of the noise $v$ are calculated (i.e. an unbiased estimate of its variance), assuming Gaussian noise.

2. Choice of a set of basis functions $\mathcal{B}$. 
3. Estimation of the parameter $\theta$ and the random walk process $\Lambda$ in eqn.(5): $\widehat{\hat{\mathrm{G}}}_{\mathrm{k}}=\mathcal{B} \theta+$ $\mathcal{B} \bar{\theta} \Lambda+v_{k}$, based on the frequency function point estimates $\hat{\mathrm{G}}_{\mathrm{k}}$. This is usually performed according to the following procedure:

(a) Estimation of $\theta$ (called $\hat{\theta}$ ), based on the knowledge of $\hat{\mathrm{G}}_{\mathrm{k}}$. Thus, the nominal model $\hat{\mathrm{G}}_{0}=\mathcal{B} \hat{\theta}$ in eqn.(4) is the least squares estimate approximation of $\hat{\mathrm{G}}_{k}$ in the subspace spanned by basis functions $\mathcal{B}$.

(b) As model error parameterization $\bar{\theta}$, the estimate $\hat{\theta}$ is chosen (this is a typical choice, when no a priori information on unmodeled dynamics is available). Thus, eqn.(5) becomes: $\hat{\mathrm{G}}_{\mathrm{k}}=\mathcal{B} \hat{\theta}(1+\Lambda)+v_{\mathrm{k}}$.

(c) Computation of an unbiased estimate of the variance of the random walk $\Lambda$, basically using an unbiased estimate of the variance of the noise, see [10] for closed form expressions and technical details. Here, the random walk is a model whose variance of the frequency response increases linearly with frequency up to a certain roll off.

(d) Quantification of the model error $\mathcal{B} \bar{\theta} \wedge$ for any frequency, i.e. calculation of its statistical properties.

A variant of the random walk for resonant systems (integrated random walk) is described in [2]: here, the error increases quadratically with the frequency, in order to produce tighter error bounds at low frequencies in the presence of resonant poles.

Step 2 contains the crucial choice of the set of (orthonormal) basis functions. In the simple case of a Laguerre expansion, one can employ the minimization of the average modeling error, as suggested in $[1,2]$. At any frequency $\omega_{j}$, the total modeling error is given by $G_{e}\left(\omega_{j}\right):=$ $\mathcal{B}\left(\omega_{j}\right) \hat{\theta}-G\left(\omega_{j}\right)$. For a closed form expression of $G_{e}\left(\omega_{j}\right)$ and its covariance $\Sigma_{e}\left(\omega_{j}\right)$, the reader is referred to [11]. We note that, for different choices of the pole in the Laguerre expansion, the covariance matrix is a function of the Laguerre pole as well: $\Sigma_{e}\left(\omega_{j}\right)=\Sigma_{e}\left(\omega_{j}, p_{i}\right)$. Therfore, we define the average modeling error (over a certain finite frequency grid $\Omega$ ) for a fixed Laguerre pole as

$$
\sum_{\omega_{j} \in \Omega} \operatorname{trace}\left(\Sigma_{e}\left(\omega_{j}, p_{i}\right)\right)
$$

The "optimal" Laguerre pole $p_{i}^{*}$ is the one that minimizes the mean error (6).

\section{Model Error Modeling}

In this section, the basic ideas of model error modeling are described. A thorough treatment of this approach is given in [15], where prediction error methods are used to calculate nominal model as well as error model. The idea described there, however, is much more general and does not depend on a certain estimation method, as we will see in the sequel. 
Let $\left(u_{m}, y_{m}\right)$ be a collection of $i / o$ measurements and assume that a nominal model $G_{n}$ of the system that generated the data has been estimated, according to some identification procedure. Then, the model error modeling strategy can be summarized as follows.

1. Compute the residual $\epsilon=y_{m}-G_{n} u_{m}$.

2. Consider the "error" system, with input $u_{m}$ and output $\epsilon$, and identify a model $G_{e}$ for this system. This is an estimate of the error due to undermodeling, the so-called model error model. Standard statistical properties of the estimated model lead to the definition of the uncertainty region of the model error (e.g., given by the $99 \%$ confidence region of the estimated model error $G_{e}$ ).

3. The uncertainty region of the nominal model is obtained by adding up the nominal model and the uncertainty region of the model error.

4. Model validation: the nominal model is not falsified if and only if it lies inside its own uncertainty region or, equivalently, if and only if zero is an element of the model error uncertainty region. This can be easily checked by looking at the corresponding uncertainty bands in the Bode or Nyquist plots.

Identification of the model error from residual data provides a separation between noise and unmodeled dynamics. In fact, $\mathrm{G}_{e}$ can be seen as an estimate of the dynamic system $\Delta(\cdot)$ (possibly nonlinear, time-varying, etc.), such that

$$
\epsilon=\Delta(u)+v
$$

where $v$ is the noise term, which is assumed to be uncorrelated with the input $u$.

A few observations can be made.

- The model error $G_{e}$ must be selected among a pre-specified class of models. Unfortunately, there is not a standard procedure for selecting the structure of the model error. This is rather based on a priori knowledge on the system generating the data, and on the purposes for which the model uncertainty is estimated. For example, a typical requirement is that unmodeled dynamics is captured at those frequencies that are significant for control design purposes.

- The size of the uncertainty region clearly depends on the selected nominal model. When a severe undermodeling occurs (due for example to the fact that we are required to deliver a low order nominal model to the robust control designer), one cannot expect the model error uncertainty region to be small at all frequencies, i.e. the nominal model will be falsified, whatever the structure of the model error is.

- A falsified nominal model may still be accepted, provided that the related uncertainty band is small at frequencies of interest.

In this paper, we will adopt the general class of linear models proposed in [16] and apply prediction error methods to both nominal model and model error model identification. 


\section{Set Membership Identification}

\subsection{Basic Ideas and connection to Model Error Modeling}

The term Set Membership Identification has been used in recent years to indicate a wide variety of robust identification techniques, that are able to handle hard bounds on the identification error. The approach considered in this paper is the one taken in [8]. In the following, we summarize its main features.

Assume that the measured $\mathrm{i} / \mathrm{o}$ data are generated by a system $S_{0}$, according to

$$
y_{m}=S_{0}\left(u_{m}\right)+v
$$

where $S_{0}$ belongs to a set $\mathcal{K}$ (the a priori information on the system) and the noise $v$ is bounded in some norm $\mathcal{Y}$, i.e. $\|v\|_{\mathcal{Y}} \leq \delta$, for given $\delta>0$. Then it is possible to define the feasible system set

$$
\mathrm{FSS}=\left\{\mathrm{S} \in \mathcal{K}:\left\|y_{m}-\mathrm{S}\left(\mathrm{u}_{\mathrm{m}}\right)\right\|_{\mathcal{Y}} \leq \delta\right\}
$$

which is the set of systems that are compatible with the measured data and the a priori assumptions. The set FSS can be very complicated, depending on the structure of $\mathcal{K}$ and the norm $\|\cdot\|_{\mathcal{Y}}$. For example, if $\mathcal{K}$ is the class of LTI systems whose impulse response has an assigned exponential decay and noise is $\ell_{\infty}$-bounded, then FSS is an infinite dimensional polytope. Obviously, for real-world systems, including nonlinearities or time-varying drifts, FSS may be much more complex.

Since the set FSS contains all the information provided by data and a priori assumptions, it is natural to evaluate the quality of a nominal model $G_{n}$ according to its worst-case error with respect to elements of FSS. In other words, the identification error associated to $G_{n}$ is given by

$$
E\left(G_{n}\right)=\sup _{S \in F S S}\left\|S-G_{n}\right\|_{\mathcal{S}}
$$

where $\|\cdot\|_{\mathcal{S}}$ is a suitable norm in the system space.

In order to identify a nominal model, a model class must be chosen. A common requirement is that the nominal model must be simple (low dimensional, linearly parameterized, etc.). Hence, a typical structure for $G_{n}$ is

$$
\mathrm{G}_{n}(\mathrm{q} ; \theta)=\sum_{i=1}^{n} \theta_{i} B_{i}(q)
$$

where the $B_{i}(q)$ are user defined basis functions, such as FIR filters, Laguerre or Kautz filters [23], generalized orthonormal basis functions [22], etc. If we denote by $\mathcal{M}$ the set of nominal models parameterized as in (10), the problem of selecting a model in $\mathcal{M}$ according to 
the criterion (9) is usually addressed as conditional set membership identification [8]. The optimal nominal model is given by the conditional central estimate

$$
\mathrm{G}_{\mathrm{n}}^{*}=\arg \inf _{\mathrm{G} \in \mathcal{M}} \sup _{S \in \mathrm{FSS}}\|\mathrm{S}-\mathrm{G}\|_{\mathcal{S}}
$$

Since in most practical situation finding an exact solution of the min-max optimization problem (11) is a prohibitive task, suboptimal estimators are considered [5]. In this paper, we will assume that noise is $\ell_{\infty}$-bounded (i.e., $\|\cdot\|_{\mathcal{Y}}=\ell_{\infty}$ ) and select as nominal model $\mathrm{G}_{\mathrm{n}}\left(\mathrm{q}, \theta^{\mathrm{r}}\right)$, where

$$
\theta^{r}=\arg \inf _{\theta \in \mathbb{R}^{n}}\left\|y_{m}-\sum_{i=1}^{n} \theta_{i} B_{i}(q) u_{m}\right\|_{\infty} .
$$

This is known as restricted projection estimate and enjoys some nice properties, including the fact that it does not depend on the actual value of the noise bound $\delta$. Here this choice is motivated only by computational reasons, as $\theta^{r}$ can be easily computed by linear programming; other more sophisticated estimation algorithms can be chosen without modifying the whole robust identification strategy.

Whatever nominal model has been identified, the actual size of the unmodeled dynamics can be evaluated from available data by exploiting the Model Error Modeling concept described in Section 3. The identification of the error system (7) can be performed via set membership identification algorithms, exploiting the noise bound on $v$. If the structure of the model error model is chosen as

$$
\mathrm{G}_{e}(\mathrm{q} ; \bar{\theta})=\sum_{i=1}^{\overline{\mathrm{n}}} \bar{\theta}_{i} \overline{\mathrm{B}}_{i}(\mathrm{q})
$$

the feasible set for the model error parameters is given by

$$
\mathrm{FES}=\left\{\bar{\theta} \in \mathbb{R}^{\overline{\mathrm{n}}}:\left\|\epsilon-\sum_{i=1}^{\bar{n}} \bar{\theta}_{i} \overline{\mathrm{B}}_{i}(\mathbf{q}) \mathrm{u}_{\mathrm{m}}\right\|_{\mathcal{Y}} \leq \delta\right\} .
$$

Then, one may select the worst-case optimal error model by computing the Chebishev center of FES

$$
\bar{\theta}^{*}=\arg \inf _{\bar{\theta} \in \mathbb{R}^{\bar{n}}} \sup _{\tilde{\theta} \in \mathrm{FES}}\|\tilde{\theta}-\bar{\theta}\|
$$

where $\|\cdot\|$ denotes the Euclidean norm (of course, other norms can be chosen).

As for (11), problem (15) may be computationally infeasible, and suboptimal solutions are sought. For example, since for $\ell_{\infty}$-bounded noise FES is a polytope in $\mathbb{R}^{\bar{n}}$, it can be recursively approximated by simpler regions, like ellipsoids or parallelotopes [4, 3], and the center of these approximating sets may be chosen as an estimate of $\bar{\theta}^{*}$.

If robust identification is oriented to $\mathcal{H}_{\infty}$ control, an uncertainty band associated to the nominal model frequency response must be delivered to the control designer. In this framework, the frequency plot of the error model uncertainty region can be obtained by mapping 
the set FES onto the complex plane for each frequency of interest. This leads to the frequency domain uncertainty set

$$
\mathcal{V}(\mathrm{FES})=\bigcup_{\omega} \mathcal{V}_{\omega}(\mathrm{FES})
$$

where

$$
\mathcal{V}_{\omega}(\mathrm{FES})=\left\{z \in \mathbb{C}: z=\mathrm{A}_{\omega} \bar{\theta}, \bar{\theta} \in \mathrm{FES}\right\}
$$

and $A_{\omega}=\left[\bar{B}_{1}\left(e^{j \omega}\right) \ldots \bar{B}_{\bar{n}}\left(e^{j \omega}\right)\right] \in \mathbb{C}^{1 \times \bar{n}}$. Once again, the computation of $\mathcal{V}($ FES $)$ may be a formidable task if the exact FES defined by (14) is considered. Hence, set approximations are useful also in this respect. Example of the resulting plots for nominal model and model error will be shown in Section 5 .

The overall set membership identification strategy can be summarized as follows.

1. Identify the nominal model $G_{n}(q ; \theta)$, using conditional estimators (12) based on the feasible system set (8).

2. Compute the residual $\epsilon=y_{m}-G_{n}(q ; \theta) u_{m}$.

3. Select a model error structure, choose the noise bound $\delta$ and compute (or approximate) the feasible model error set (14).

4. Identify a nominal model error $G_{e}(q ; \bar{\theta})$, using optimal or suboptimal estimators based on FES.

5. Map the nominal model plus the model error and its uncertainty region onto the frequency domain.

Remark. The use of residual data in set membership identification for evaluating the worst-case $\mathcal{H}_{\infty}$ norm of the unmodeled dynamics has been introduced in [9], for standard least-squares nominal models. The above set membership model error modeling strategy can be seen as a general framework in which the structures of the nominal and error models, and the corresponding identification algorithms must be chosen by the user according to the specific problem (a priori knowledge, noise bound, error norm, etc.).

In the next subsection, it will be shown how it is possible to exploit the above framework to obtain a reliable measure of the size of the unmodeled dynamics. This will also provide useful information about the selection of the nominal model class.

\subsection{Model Validation and Model Class Selection}

Lift this to a seperate section (WR+AG)?

In the Set Membership procedure previously described, a key parameter is the noise bound $\delta$ in eqn. (14). Indeed, the size of the model error uncertainty clearly depends on $\delta$. 
Hence, $\delta$ can be used as a tuning parameter, in order to evaluate the "distance to validation" of the selected nominal model.

Let us consider the set $\mathcal{V}_{\omega}(\mathrm{FES})$ in (16) and define

$$
\mathrm{d}(\omega)=\min _{z \in \mathcal{V}_{\omega}(\mathrm{FES})}|z|
$$

If we set

$$
\mathrm{d}=\sup _{\omega} \mathrm{d}(\omega)
$$

then it is clear that the nominal model can be deemed to be unfalsified if $d=0$. If ellipsoidal approximations of FES are considered, the sets $\mathcal{V}_{\omega}(\mathrm{FES})$ are ellipses in the complex plane and the optimization problem (17) can be easily solved. Then, $d$ in (18) can be approximated by taking the maximum over a finite number of frequencies in the range of interest.

It is worth observing that $d$ is a function of $\delta$, since FES (and its frequency image $\mathcal{V}_{\omega}(\mathrm{FES})$ ) depend on the noise bound. Then, one can look for the minimum value of $\delta$ for which the nominal model is still not falsified by data, i.e.

$$
\delta^{*}=\min _{\{\delta: d=0\}} \delta .
$$

Since $d$ is an increasing function of $\delta$, the computation of $\delta^{*}$ can be easily performed within the desired precision, using a standard bisection on $\delta$. Obviously, $\delta^{*}$ will not be larger than the value of the norm given in eqn. (12), which is the minimum noise bound for the nominal model when using no model error model.

It is believed that the quantity $\delta^{*}$ is important in several respects. In particular, it allows one to deliver the smallest frequency domain uncertainty region associated to the model error model, which does not falsify the nominal model. This meets a typical requirement of the control designer: the "tightest" uncertainty band around the nominal model, without contradicting the information given by data. Clearly, $\delta^{*}$ depends on the model class selected for identification of the model error model: the "richer" this class, the smaller $\delta^{*}$. On the other hand, a too simple model class may result in a large value of $\delta^{*}$, which would contradict the a priori assumption on the noise size. Hence, $\delta^{*}$ can be used to "validate" either the noise bound or the model error model class, according to the available a priori information. If a reliable a priori estimate $\hat{\delta}$ of the noise size is available, the model class for model error identification should be chosen so that $\delta^{*}$ turn out to be as close as possible to $\hat{\delta}$. Conversely, if the model error model class is suggested by a priori information about the system dynamics, $\delta^{*}$ provides a validation tool for the noise bound (for example, if $\delta^{*}$ is much smaller than the a priori bound, one may conclude that the latter was overestimated).

Another useful property of $\delta^{*}$ is that it gives a rationale for selecting an appropriate model class for nominal model identification. Let us denote by $p$ a vector of parameters that define a model class for $G_{n}$ (for example, the number and/or the pole locations of the family of basis functions $\left.B_{i}(q)\right)$. For each $p$, one can perform set membership identification of nominal 
model and model error model as suggested in Section 4.1, and compute $\delta^{*}(p)$. Then, at least in principle, one can choose the optimal model structure by solving

$$
\inf _{p} \delta^{*}(p)
$$

In practice, the optimization in (20) can be performed over a finite set $\mathcal{P}=\left\{p_{1} \ldots p_{m}\right\}$ (see the example in the next section, where $p$ is the pole of a Laguerre expansion of fixed order).

\section{Simulation Example}

\subsection{Experimental Setup}

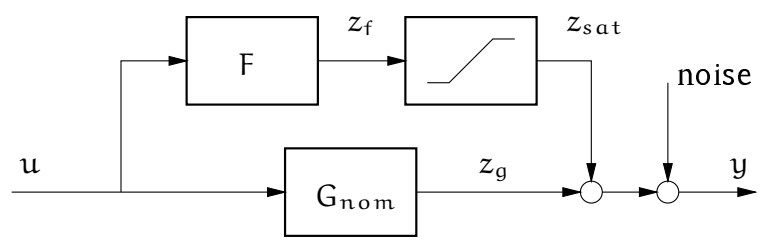

Figure 1: The experimental setup: A linear system $G_{\text {nom }}$ in parallel with a linear Filter $F$ and a saturation nonlinearity. The output signal $y$ is corrupted by noise.

Consider the experimental setup as shown in Fig. 1. Its core consists of the following fifth order linear system

$$
\mathrm{G}_{\text {nom }}(\mathrm{s})=\frac{24.15(\mathrm{~s}+1)}{0.012 \mathrm{~s}^{5}+0.25 \mathrm{~s}^{4}+1.80 \mathrm{~s}^{3}+5.86 \mathrm{~s}^{2}+7.33 \mathrm{~s}+1}
$$

Its dynamics are perturbed by a nonlinearity in the medium frequency range. This is due to a parallel connection with a linear second order filter $F(s)=\frac{2 s}{0.1 s^{2}+s+0.09}$ and a saturation nonlinearity, that saturates at level $l=1.2$. According to the amplitude curve of the filter, which is given in Fig. 2 , the nonlinearity will be "active" in the frequency range $\omega=[0.1 ; 10]$, dependent on the amplitude of the input signal. The system will behave linearly, when the output of the filter $z_{f}$ is smaller than 1.2 in amplitude; then $y=z_{g}+z_{\text {sat }}=\left(G_{\text {nom }}+F\right) u$ holds.

In order to be able to apply Stochastic Embedding, the input signal we use is a linear combination of 53 sinusoids(the contained frequencies are marked as crosses in Fig. 4). We use a sample time of $T_{s}=0.04 \mathrm{~s}$ and 3000 samples. We will use four different data sets to examine the behavior of the linear identification methods to this nonlinear plant, which are shown in the following table: 


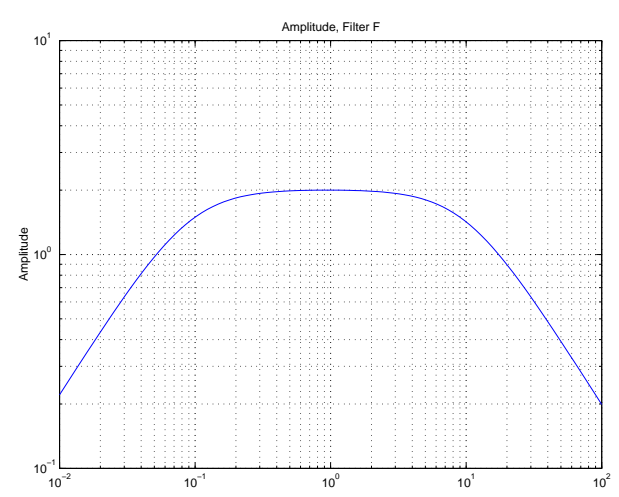

Figure 2: Amplitude plot of the second order linear filter F.
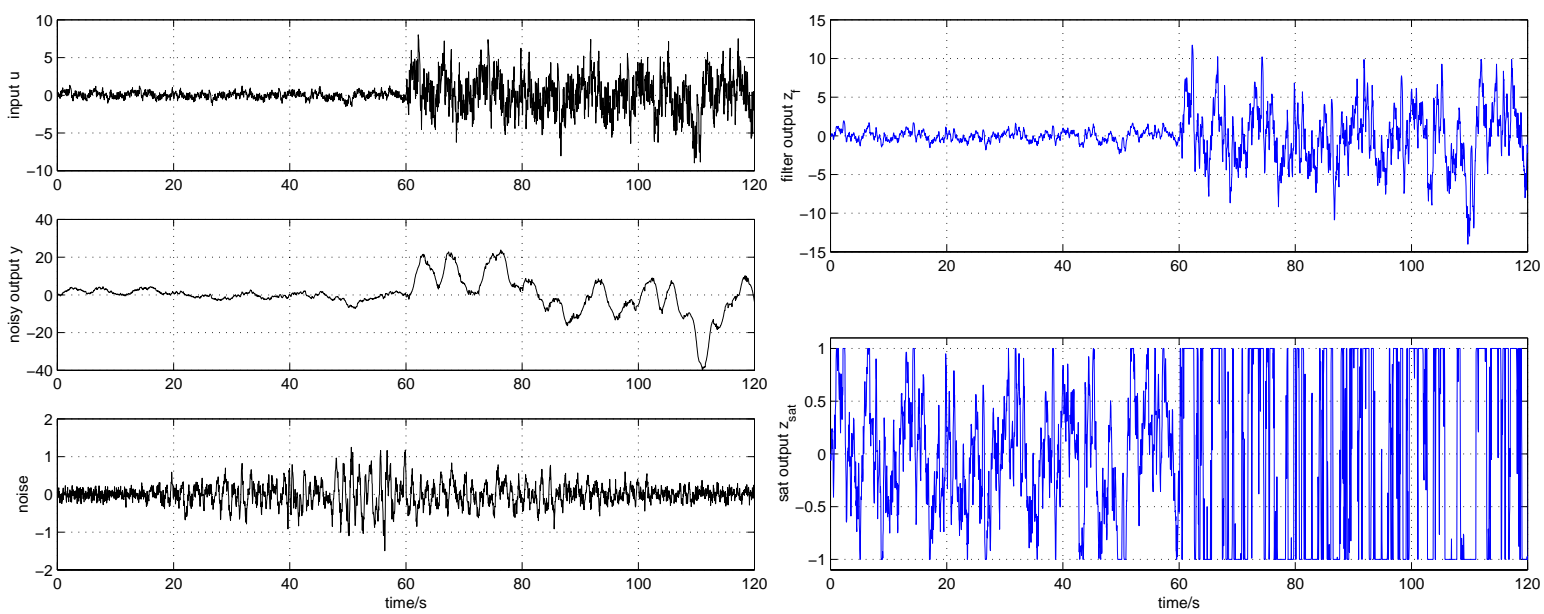

Figure 3: Selected signals from dataset 3. Left: Test input $u$, noisy output $y$ and output error noise (top-down). Right: Output of the filter $F, z_{f}$, and of the saturation nonlinearity, $z_{\text {sat }}$.

\begin{tabular}{c|lcc} 
Dataset & excitation of saturation & input level (first half) & input level (sec. half) \\
\hline 1 & none (linear behavior) & 1 & 1 \\
2 & low & 1 & 2 \\
3 & medium & 1 & 6 \\
4 & high & 10 & 10
\end{tabular}

Experiments 1 and 4 excite the system with an input signal as described above with an amplitude of about 1 and 10 respectively. These input signals will lead to an either linear or completely nonlinear behavior. Experiments 2 and 3 switch from a low amplitude input signal to a higher one. We note that the results obtained later on will not change significantly when switching form a higher to a lower amplitude. For experiment 3, these signals are reported in Fig. 3.

First, we perform spectral analysis using the noiseless output signal $y$ and the corre- 

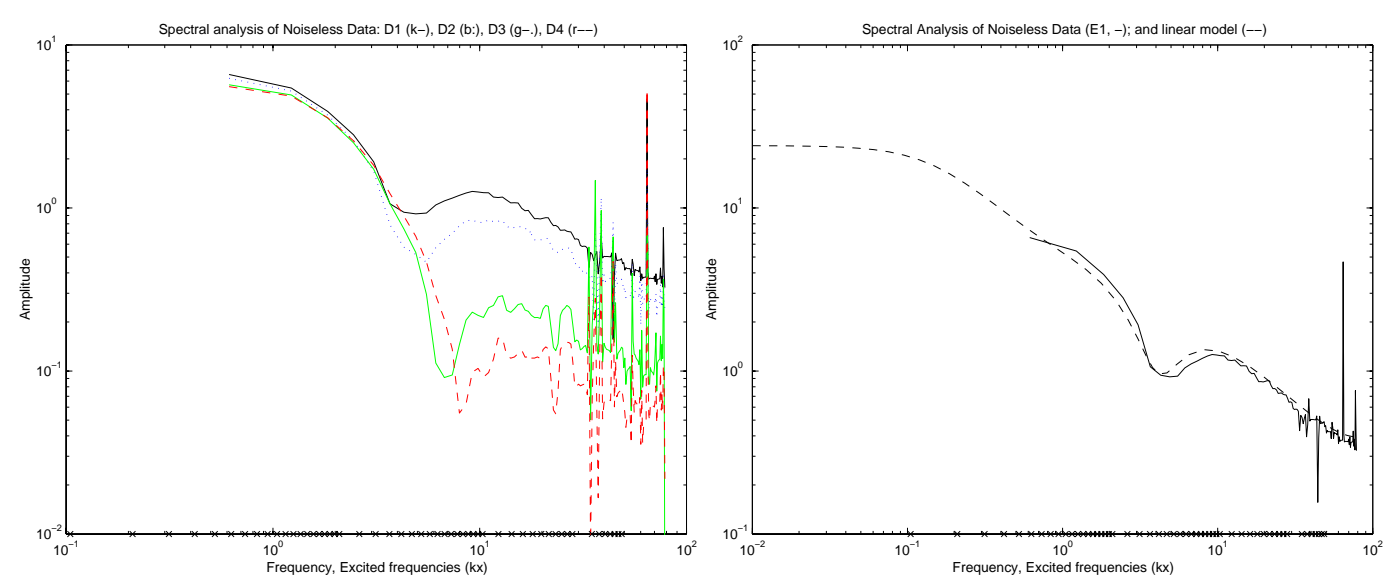

Figure 4: Left: Spectral analysis (employing a small smoothing window) of the four noiseless datasets: dataset 1 (solid), dataset 2 (dotted), dataset 3 (dash-dotted) and dataset 4 (dashed). The crosses on the frequency axis mark those 53 frequencies, contained in the multi-sinusoid input signal. Right: Bode plot of the linear "mode" $G_{\text {nom }}+F$ (dashed) in comparison to the spectral analysis of dataset 1 (solid).

sponding input signal $u$. The result is depicted in Fig. 4 (left), together with the Bode plot of the linear system $G+F$, which will be "the true plant" for low input level (right). All frequency profiles are quite similar for low frequencies, while they are significantly different at higher frequencies. Clearly, the frequency measurements obtained in this case may change for different input signals, especially for an input signal with a completely different power spectrum. Nevertheless, we keep the obtained frequency profiles as a reference in the comparison of the different methods, for the specific inputs selected.

Add something about 2nd moment equivalent (LL)

Additionally, the output is corrupted by noise, which is also shown in Fig. 3 (left). The noise is a normally distributed one with variance 0.0102 , added to a scaled version (to a maximum amplitude of about 1.4) of the $200 \mathrm{~Hz}$ signal registered during the Loma Prieta earthquake in the Santa Cruz Mountains in October 1989 (which has been measured at the Charles F. Richter Seismological Laboratory and made been available by The MathWorks Inc.). This noise signal has been chosen in order to avoid usage of standard stationary stochastic processes or boundary visiting signals as noise models.

In the following, datasets 1-4 will be used for identification purposes. Different datasets (obtained by adding a different output noise) will be employed in the model validation step, for those methods that allow an explicit validation procedure (i.e. Model Error Modeling and Set Membership Identification).

The goal of the identification experiments is to obtain a nominal model of order not larger than 4 with a reliable uncertainty description in the frequency domain, which is of suitable 


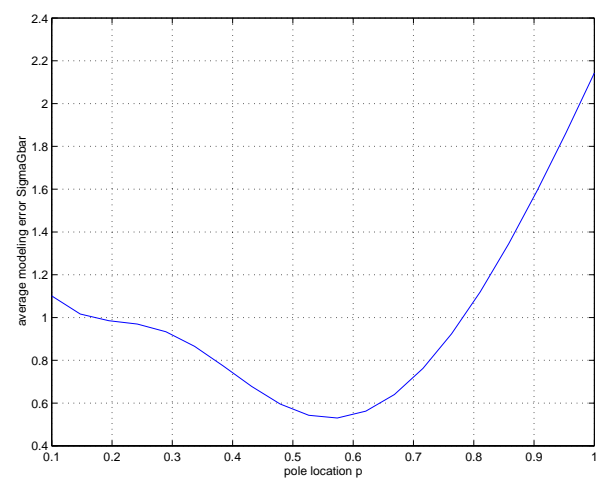

Figure 5: NSSE: Laguerre pole vs. mean error over all frequencies, in order to choose the "optimal" Laguerre pole, that guarantees minimum error. Result is based on dataset 4.

size for robust controller design using standard $\mathcal{H}_{\infty}$ methods.

\subsection{Identification via Non-Stationary Stochastic Embedding}

The first step within non-stationary stochastic embedding is the estimation of the transfer function at those frequencies contained in the input signal. The second step is then the estimation of the nominal model, based on these transfer function point estimates. By problem definition we are restricted to models of order 4, thus we choose such a Laguerre expansion for our identification. In order to obtain comparable results, we use a confidence level of $99 \%$ here as well as for the prediction error methods in the Model Error Modeling approach. We remark, however, that the shape of the uncertainty band does not change paramountly at low and medium frequencies, when decreasing the confidence level. Moreover, preliminary test runs show that the integrated random walk will produce uncertainty bands that are quite tight for lower frequencies and useless large for higher frequencies, whereas the random walk (increasing linearly with frequency) produces reasonable uncertainty bands. Therefore, we conclude that the system does not contain important resonances, and continue with errorpropagation by random walk strategy. We are left with the choice of the pole in the Laguerre basis. Therefore, we employ the minimization of the average modeling error, as suggested in Sec. 2. A typical plot of mean error vs. pole location for fixed order is reported in Fig. 5 (dataset 4).

Choosing pole for the Laguerre expansion to $p=-0.2895$ (dataset 1,2 ) and $p=-0.5737$ (dataset 3,4 ) respectively, least squares estimation of the nominal model and error propagation leads to the results as depicted in Fig. 6 . Note, that choice of error propagation and confidence level do not influence the nominal model. In all four cases, the uncertainty band includes the frequency profiles obtained by spectral analysis quite well. 

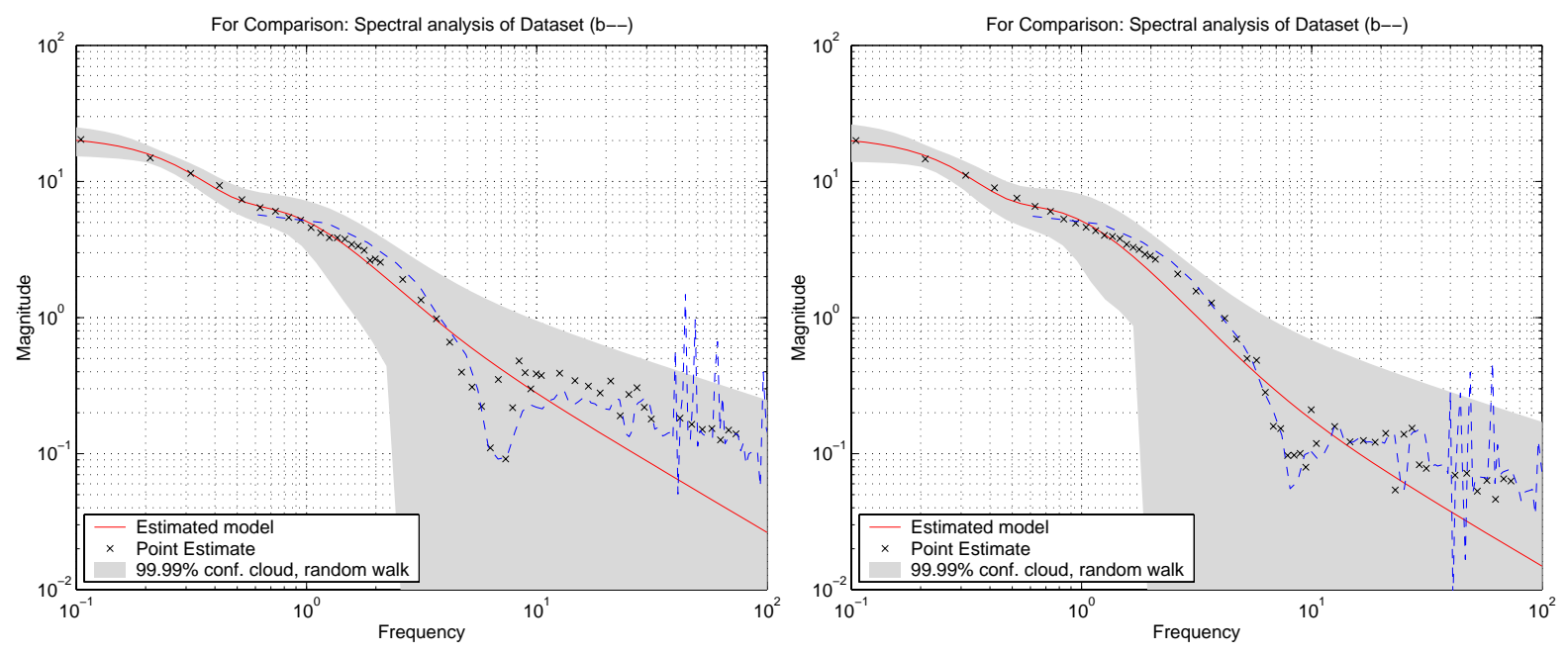

Figure 6: NSSE: Identification based on datasets 3 (left) and 4 (right) respectively. Both approaches use a 4 th order Laguerre expansion with pole located at $p=-0.5737$ and random walk as error propagation. The $99 \%$ confidence clouds are drawn (shaded), as well as transfer function point estimates (crosses), estimated nominal model (solid) and spectral analysis of noiseless data (dashed).

\subsection{Identification via Prediction Error Methods}

We estimate an Output Error model of order 4 as nominal model using prediction error methods. The result for dataset 1 , together with the standard residual test, is reported in Fig. 7 . The real plant is clearly not in the confidence region, which is rather small due to the large amount of data (3000 samples) used here. Moreover, the model does not seem to pass the classical residual test. A similar statement holds for the remaining three datasets. Hence, we are in need of more detailed information about the accuracy of the identified 4rth oder OE model. Based on the obtained nominal model, we therefore proceed with estimation of an error model based on the residual data (calculated from the validation dataset). A crucial step is to choose the structure of the error model, so that the nominal model is not falsified by its own error model, i.e. zero has to be inside the, say $99 \%$, confidence region of the error model.

We choose a error model of the form

$$
A(q) \epsilon(t)=\frac{B(q)}{F(q)} u\left(t-n_{k}\right)+\frac{C(q)}{D(q)} v(t)
$$

with polynomial orders $n_{A}=1, n_{B}=9, n_{C}=n_{D}=5, n_{F}=10$ and delay $n_{k}=1$ for validation dataset 1 . The result is reported in Fig. 8 (left). The model error model validates the nominal model, at the price of a larger confidence region (compared to the one in Fig. 7).

We follow the same reasoning for validation dataset 4 . As we face non-trivial undermodeling due to a low order nominal model and a highly non-linear behavior, it is not straight- 

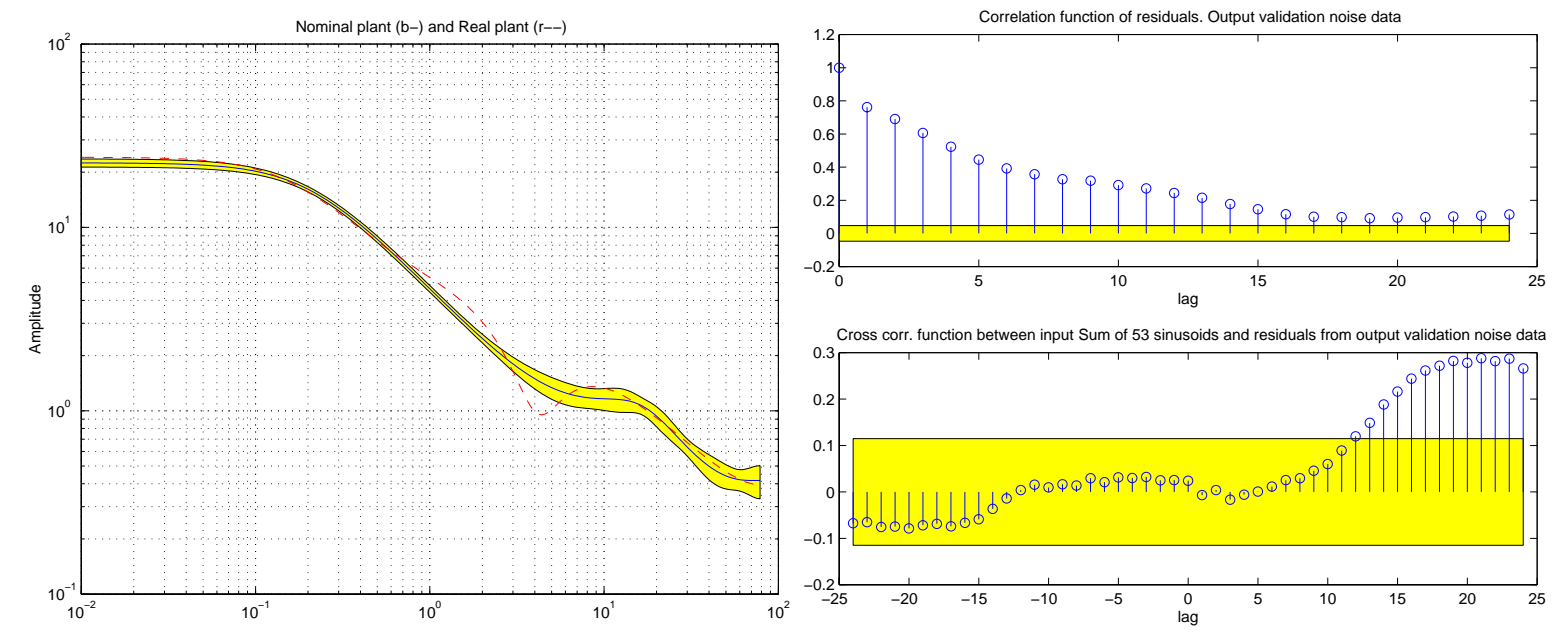

Figure 7: PEM: Left: 4rth order output error model, using dataset 1 for identification. The 99\% confidence region is given as well (shaded). Right: Correlation function of the residuals (upper plot) and cross-correlation function (lower plot) using the validation dataset.

forward how to choose order and structure of the model error model in order to validate the nominal model. After several trials, we used an error model with polynomial orders $n_{A}=4, n_{B}=9, n_{C}=n_{D}=5,=n_{F}=10$ and delay $n_{k}=1$, which does not verify the nominal model, but gives better information on the frequency range where the error occurs. The result is reported in Fig. 8 (right).

\subsection{Identification via Set Membership Estimation}

To obtain a 4rth order nominal model we use a 4rth order Laguerre expansion. The poles of the Laguerre expansion are chosen according to the strategy suggested in Section 4.2. Hence we will use the hard bound on the noise as additional tuning parameter for the selection of the model class. A typical picture of Laguerre pole location vs. minimum, non-falsifying noise bound is reported in Fig. 9.

Using dataset 1,3 and 2,4, we end up with a Laguerre pole at $p=0.97$ and $p=0.98$ respectively. Due to the increasing amount of nonlinearity, the minimum, non-falsifying error bounds increase by $\delta=0.70,0.83,1.29,2.63$ for datasets $1-4$ respectively. These "identified" noise bounds for datasets 1-3 coincide quite well with the size of the actual noise of about 1 , as given in Fig. 3. Clearly, the higher value for dataset 4 is due to a significant contribution from the nonlinearity.

In order to obtain a frequency domain uncertainty representation, we follow the procedure suggested in Section 4.2. To reduce the computational burden, we approximate the FES in (14) via ellipsoids [4]. Fig. 10 reports the final result for datasets 1 and 4. We observe, that the nominal model matches the linear model quite well in the low and middle frequency 

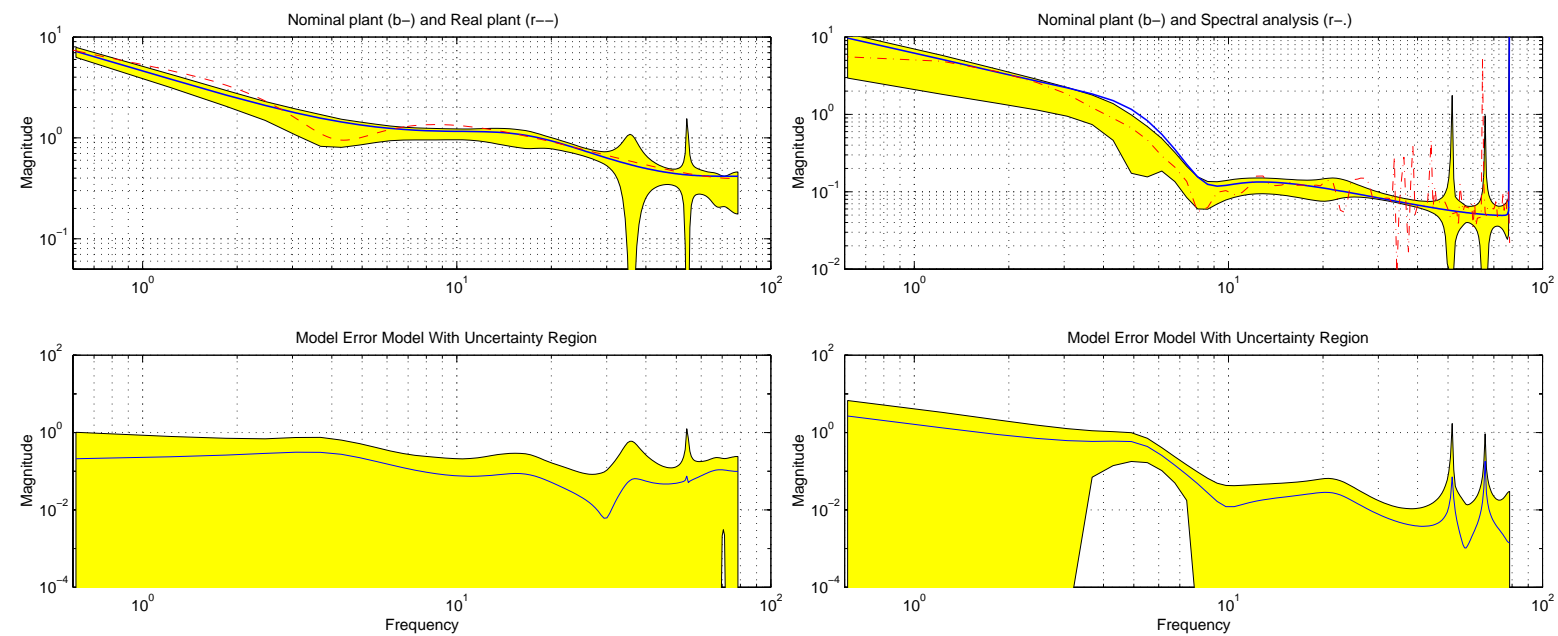

Figure 8: PEM: Left: 4rth order output error model, using dataset 1 for identification. Model error modeling with a $(1,9,5,5,10,1)$-error model using validation data set 1 . The real plant is shown as well (dashed). Right: 4rth order output error model, using dataset 4 for identification. Model error modeling with a $(4,9,1,1,11,2)$-error model using validation data set 4. Upper plots: nominal model (solid) along with uncertainty region (shaded) and spectral analysis of noiseless data (dashed). Lower plots: model error models with uncertainty regions.

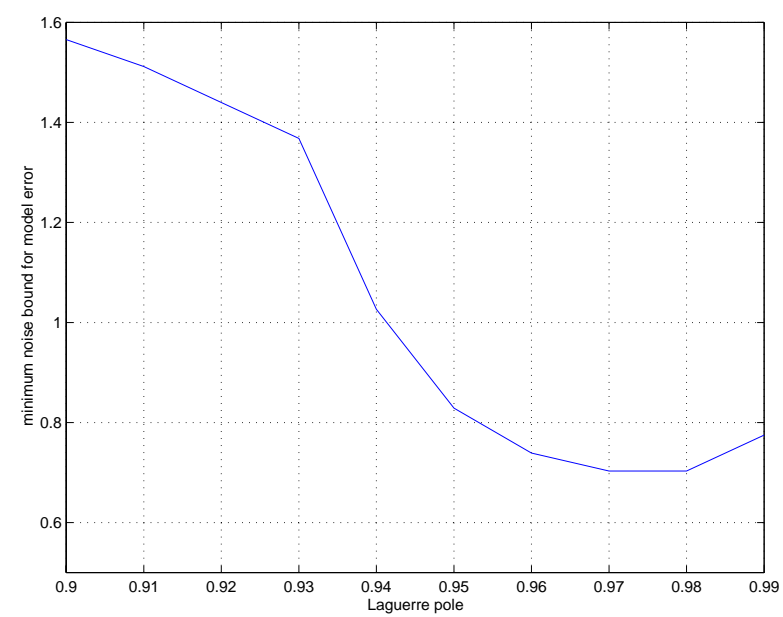

Figure 9: SMI: Location of Laguerre pole versus minimum, non-falsifying noise bound of the model error (dataset 1 ). 

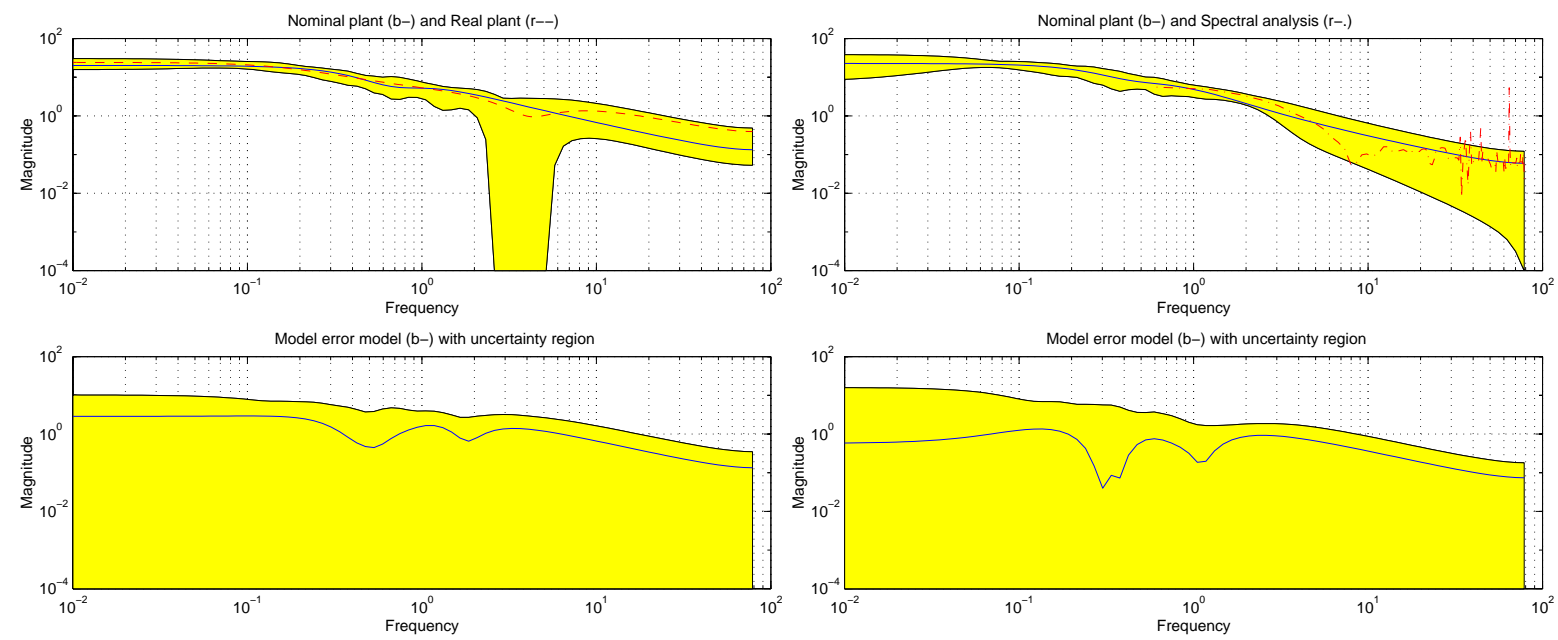

Figure 10: SMI: For datasets 1 (left) and 4 (right): The upper plot shows restricted projection estimate (solid) inside the unfalsified uncertainty region (shaded) compared to linear plant (left plot) and spectral analysis of noiseless data (right plot) respectively. The lower plots show the model error model inside its unfalsified uncertainty region.

ranges. Moreover, the uncertainty band is reasonably small and includes the frequency profiles provided by spectral analysis.

\section{5}

Why the comparison in the next subsection?

(LL)

\subsection{Comparison}

The system generating the data (see Fig. 1) contains a saturation, which is an odd static nonlinearity with a gain varying between 0 and 1 . We therefore compare the uncertainty regions, delivered by identification with the magnitude envelope given by $G_{\text {nom }}$ and $\left(G_{\text {nom }}+F\right)$ as lower and upper bound respectively. For ease of notation, we will refer to the latter region as the "true uncertainty region". Figures 11 and 12 report the results for datasets 2 and 4 respectively. The identified uncertainty regions should cover parts of the true uncertainty region, obviously depended of the excitation of the nonlinearity by the input signal.

We observe, that for both datasets the uncertainty set produced by prediction error methods is misleadingly tight, because of a large amount of data (1500 samples). Moreover, the nominal model is falsified for the medium frequency range for dataset 4, see Fig. 8.

Moreover, we note that for dataset 4, Fig. 12, preferably the "lower part" of the true uncertainty region is captured by all methods. This is clear due to the lower gain of the nonlinearity in this case (as the level of the input signal is higher). 

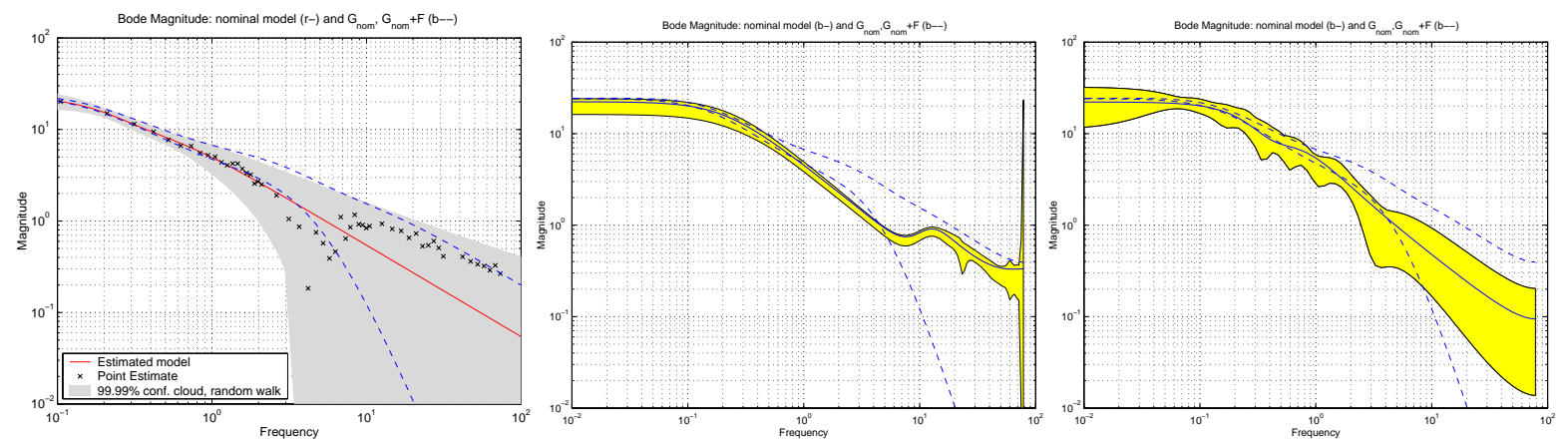

Figure 11: Dataset 2: Nominal model (solid) and uncertainty region (shaded) in comparison to the amplitudes of $\left(\mathrm{G}_{\text {nom }}+\frac{1}{2} \mathrm{~F}\right) \pm \frac{1}{2} \mathrm{~F}$ (dashed). From left to right for NSSE (continuous time), MEM and SMI (discrete time).
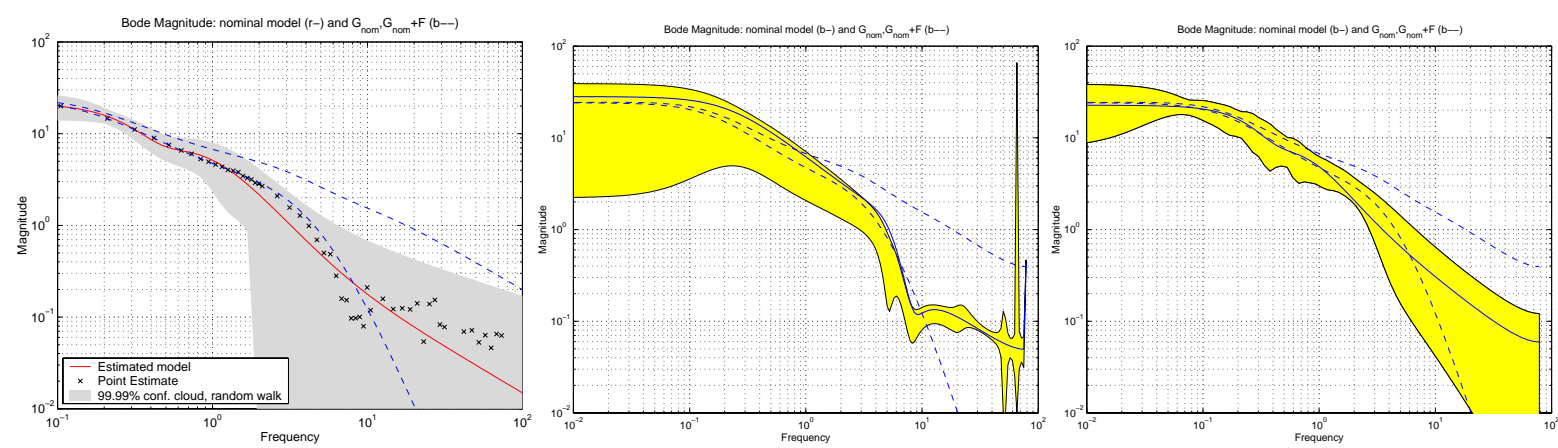

Figure 12: Dataset 4: Nominal model (solid) and uncertainty region (shaded) in comparison to the amplitudes of $\left(\mathrm{G}_{\text {nom }}+\frac{1}{2} \mathrm{~F}\right) \pm \frac{1}{2} \mathrm{~F}$ (dashed). From left to right for NSSE (continuous time), MEM and SMI (discrete time). 


\subsection{Properties and benefits of the three methods}

Our comparison addresses three different approaches to robust identification: Stochastic Embedding, Model Error Modeling and Set Membership Identification. The first two approaches have been developed in the statistical framework, while the latter relies on UBB error assumptions. Due to the different nature of the three methods, an overall comparison might become unfair at a certain stage. Due to restrictions on harmonic input signal for example, one might not always be able to apply Stochastic Embedding to a given dataset. We therefore concentrate ourselves on highlighting the most important properties and benefits of each method.

Model Error Modeling and Set Membership Identification allow an explicit validation step (on fresh validation data). As no further knowledge than the nominal i/o behavior is needed, these frameworks also allow validation of an already existing model for the process. Even when invalidated, valuable information on the underlying error can be obtained, for instance in which frequency range the model is sufficiently reliable.

In contrast, Stochastic Embedding allows the choice between different random walk models (the variance may increase in different ways with frequency) to perform the error propagation. Although this is no explicit validation step, it is a quite reasonable concept to produce reliable confidence regions.

The Model Error Modeling setup enjoys flexibility in the choice of the structure of the nominal model, to end up with for instance OE or ARX models. In contrast, Stochastic Embedding and the presented Set Membership Identification are (the latter at least in this context) tied to a parameterization of orthonormal basis functions. Both schemes, however, come along with a rationale for choice of the pole in a Laguerre expansion. It is worth to note that both end up with basically the same recommendation (in our case at $s=-0.29,-0.57$, corresponding to $z=0.98,0.97$ in discrete time).

A remaining problem in the model error modeling approach is the correct order and structure selection of the model error model, which is in general not straightforward. This problem has also been reported in [21], where the authors suggest an adaptive and nonparametric frequency-domain method that estimates the frequency response of the model error and also allows local tuning in different frequency bands.

We observe, that all methods deliver an estimated nominal model, along with an uncertainty region, which is certainly suited for robust controller design, for instance using an $\mathcal{H}_{\infty}$ mixed sensitivity approach, based on the estimated uncertainty band in the frequency domain.

\section{Conclusions and Future Works}

We compared identification methods, working in the time and frequency domain and following statistical or deterministic philosophy. Due to these different natures of the approaches, 
a fair comparison is quite difficult. Moreover, application of one of the methods will heavily depend on the a priori knowledge (for example, if harmonic input signals are possible or a priori information on the noise amplitude or statistics are available). However, we showed the main features of the methods and obtained reliable nominal models and acceptable related uncertainties in all three cases.

$$
\text { Some ' 'nonlinear', comments (LL) }
$$

Moreover, the ideas of model error modeling and their application in the context of set membership identification have been analyzed. In particular, it has been shown that the separation of noise and unmodeled dynamics is quite natural in this framework, and that the minimum noise bound for which a nominal model is not falsified by the data can be easily computed and used as a tool for model class selection.

Complementary work has still to be done, in several directions. For example, more complicated model error model structures are needed, to cope with nonlinearities or time-varying drifts. Obviously, this requires more sophisticated identification algorithms and smarter approximations in the computation of the uncertainty regions. Moreover, accurate criteria for the a priori selection of the structure of the model error model, depending on the specific control-oriented identification problem, must still be investigated.

\section{References}

[1] J. H. Braslavsky. Model error quantification via non-stationary stochastic embedding: Hairdryer example. CIDAC, Dept of Electrical Engineering, Univ of Newcastle, Callaghan, Australia. Manual for NSSE-package, Mar. 1999.

[2] J. H. Braslavsky and G. C. Goodwin. A note on non-stationary stochastic embedding for modelling error quantification in the estimation of resonant systems. Technical Report EE99014, CIDAC, Dept of Electrical Engineering, Univ of Newcastle, Callaghan, Australia, Jan. 1999.

[3] L. Chisci, A. Garulli, A. Vicino, and G. Zappa. Block recursive parallelotopic bounding in set membership identification. Automatica, 34:15-22, 1998.

[4] E. Fogel and F. Huang. On the value of information in system identification - bounded noise case. Automatica, 18(12):229-238, Dec. 1982.

[5] A. Garulli, B. Z. Kacewicz, A. Vicino, and G. Zappa. Error bounds for conditional algorithms in restricted complexity set membership identification. IEEE Trans. on Automatic Control, 45(1):160-164, 2000. 
[6] A. Garulli and W. Reinelt. On model error modeling in set membership identification. In Proc. of the System Identification Symposium SYSID, pages WeMD1-3, Santa Barbara, CA, USA, June 2000 .

[7] A. Garulli, A. Tesi, and A. Vicino, editors. Robustness in Identification and Control. Number 245 in Lecture Notes in Control and Information Sciences. Springer-Verlag, 1999.

[8] A. Garulli, A. Vicino, and G. Zappa. Conditional central algorithms for worst-case set membership identification and filtering. IEEE Trans. on Automatic Control, Jan. 2000.

[9] L. Giarrè, M. Milanese, and M. Taragna. $\mathcal{H}_{\infty}$ identification and model quality evaluation. IEEE Trans. on Automatic Control, 42(2):188-199, 1997.

[10] G. C. Goodwin. Identification and robust control: Bridging the gap. In Proc. of the 7th IEEE Mediterranian Conference on Control and Automation, Haifa, Israel, June 1999.

[11] G. C. Goodwin, J. H. Braslavsky, and M. M. Seron. Non-stationary stochastic embedding for transfer function estimation. In Proc. of the 14th IFAC World Congress, Bejing, China, July 1999.

[12] G. C. Goodwin, M. Gevers, and B. Ninness. Quantifying the error in estimated transfer functions with application to model order selection. IEEE Trans. on Automatic Control, 37(7):913-928, July 1992.

[13] R. L. Kosut, G. C. Goodwin, and M. P. Polis. Special issue on system identification for robust control design. IEEE Trans. on Automatic Control, 37(7), July 1992.

[14] L. Ljung. Comments on model validation as set membership identification. In A. Garulli, A. Tesi, and A. Vicino, editors, Robustness in Identification and Control, pages 7-16. Springer, 1999.

[15] L. Ljung. Model validation and model error modeling. In Proc. of the Aström Symposium on Control, pages 15-42, Lund, Sweden, Aug. 1999. Studentliteratur, Lund, Sweden.

[16] L. Ljung. System Identification - Theory For the User. PTR Prentice Hall, Upper Saddle River, N.J., 2nd edition, 1999.

[17] M. Milanese, J. P. Norton, H. Piet-Lahanier, and E. Walter, editors. Bounding Approaches to System Identification. Plenum Press, New York, 1996.

[18] M. Milanese and A. Vicino. Optimal estimation theory for dynamic systems with unknown but bounded uncertainty: an overview. Automatica, 27(6):997-1009, June 1991. 
[19] W. Reinelt, A. Garulli, L. Ljung, J. H. Braslavsky, and A. Vicino. Model error concepts in identification for control. In Proc. of the 38th IEEE Conference on Decision and Control, pages 1488-1493, Phoenix, AZ, USA, Dec. 1999.

[20] T. Söderström and K. J. Åström. Special issue on trends in system identification. Automatica, 31(17), Dec. 1995.

[21] A. Stenman and F. Tjärnström. A nonparametric approach to model error modeling. In Proc of the IFAC Symposium on System Identification SYSID, Jun 2000. Accepted.

[22] P. M. J. Van den Hof, P. S. C. Heuberger, and J. Bokor. System identification with generalized orthonormal basis functions. Automatica, 31(12):1821-1834, 1995.

[23] B. Wahlberg. System identification using Laguerre models. IEEE Trans. on Automatic Control, 39:1276-1282, 1991.

[24] B. Wahlberg and L. Ljung. Hard frequency-domain model error bounds from leastsquares like identification techniques. IEEE Trans. on Automatic Control, 37(7):900912, 1992.

[25] E. Walter and H. Piet-Lahanier. Estimation of parameter bounds from bounded-error data: a survey. Mathematics in Computer and Simulation, 32:449-468, 1990. 